\title{
Coal Mine Disaster Prediction
}

\author{
Harshitha.P ${ }^{1}$, Hiranmayee S Dixith ${ }^{2}$, Krithi $^{3}$, Neha Rajeeva ${ }^{4}$, Dr. Shashikala ${ }^{5}$ \\ 1,2,3,4 UG Student, Information Science and Engineering, BNMIT, Bangalore \\ ${ }^{5}$ Professor, Head of Dept. of Information Science and Engineering, BNMIT, Bangalore, India.
}

\begin{abstract}
Coal may be an exceptionally imperative common asset to our nation. There are numerous perilous conditions like increment in temperature and mugginess, discharge of hurtful gasses. These conditions make an unsafe environment for specialists to work in and posture a hazard to their lives. Hence security of the specialist in a coal mine has ended up a genuine issue to be tended to. It is very troublesome to screen all environment conditions ceaselessly in a coal mine by human creatures. There can be a duty-bounded set-up with the technique to feel the original atmosphere of the mine and determine whether it matches for a human being's presence. In the event that not, the framework ought to alarm the laborer around the threat. That way the framework should be able to help dodge the devastation. To serve this reason ready to combine IoT and Machine Learning Forecast procedures such as Ensemble Learning to ceaselessly take the test set from the coal mine environment and utilize history of conditions to foresee the threat is created.
\end{abstract}

Key Words: Coal Mines, IoT, Machine Learning, Ensemble Learning, SVM, Random Forest, KNN.

\section{INTRODUCTION}

Coal mining process deals with extracting coal from the ground. Coal is valued for its energy content and since 1880s, it has been widely used to generate electricity. The most significant uses of coal are in generation of electricity, steel production, cement manufacturing and as a liquid fuel and many industrial applications. It is said that coal can offer assistance in a noteworthy sum of financial development. Future of India in the energy department and its prosperity are integrally dependent upon mining and using coal, its most abundant, affordable and dependent energy supply. But there are many hazardous conditions like increase in temperature and humidity, leak of noxious gases such as hydrogen sulphide or hazardous characteristic gases such as methane, tidy blasts, collapsing of mine stops, mining-induced seismicity, flooding. These conditions make an unsafe environment for laborers to work in and create a risk to their lives. This is making numerous issues in accessibility of laborers for the coal mining industry. It is quite difficult to monitor all environment conditions continuously in a coal mine by human beings. Underground coal mining includes the next hazard than open pit mining due to the issues of ventilation and potential for collapse. In any case, the utilization of overwhelming apparatus and the strategies performed amid unearthing result into security dangers in all sorts of mining. Present day mines regularly actualize a few security strategies, instruction and preparing for laborers, wellbeing and security benchmarks, which lead to considerable changes and security level both in opencast and underground mining. Thus, safety of the worker in a coal mine has become a serious issue to be addressed.

\section{RELATED WORKS}

Since ages, the visit coal mine security mishaps have caused genuine casualties and tremendous financial misfortunes. It is pressing for the worldwide mining industry to extend operational effectiveness and progress generally mining security. Numerous papers have proposed person thoughts about the IoT Setup and the Prediction Framework which gave an inspiration to this paper. Coal Mining Safety using IoT to monitor the temperature, humidity, gas and status of smoke in an underground mine uses IoT with wireless sensor network (WSN). The major advantage of this work is it detects the conditions dynamically and alarms the worker for safety and can improve the level of monitoring production safety and reduce accident in the coal mine. The work is limited to alarming during time of danger. The miners have less chances of getting out of the mines. If the danger can be predicted before, it will be easy to take precautions [1]. Prediction of Coal /Gas Outbursts based on selective ensemble learning to predict the coal or gas outbursts had component learners consisted of RS-PNN network, and the redundant component learners were removed from the ensemble learners using a ensemble learning algorithm based on variable similarity cluster technology, and voting to the retained based learners was used as the output of the ensemble learners. It uses Selective ensemble learning. The major advantage of this work is effectively improved the diversity of component learners a generalization performance of ensemble learners. The work is limited to guarantee the generalization capability [2]. Application of Support Vector Machine in Coal and Gas Outburst Area Prediction to predict the coal and gas outburst have the dominant factors where the input vectors and the degree of outburst danger is divided into four types. It uses Support vector machine (SVM) based on statistical learning theory (SLT). The major advantage of this work the multiclass SVM classifier, trained with the sampling data, identifies out the four types of coal and gas outburst states. The work is limited to optimizing kernel function and taking the dataset [3].

\subsection{Ensemble Learning:}

\section{METHODOLOGY}

The yield of the past module is encouraged as an input to this module. In measurements and machine learning, outfit strategies utilize numerous learning calculations to get better predictive performance than may well be gotten from any of the constituent learning calculations alone. Not at all like a factual outfit in measurable mechanics, which is ordinarily boundless, a machine learning gathering comprises of as it were a concrete limited set of elective models, but regularly permits for much more adaptable structure to exist among those alternatives. The 
outfit strategy utilized here is Max Voting. In this method, different models are utilized to form expectations for each information point. The expectations by each sub classifier is considered as a 'vote'. The forecast which we get from the lion's share of the models are utilized as the extraordinary desire. The calculations to create the classification outline are recorded underneath.

Our ensemble learning algorithm employs the following algorithms for forecast:

\subsubsection{K-Nearest Neighbors}

Definition: Neighbors based classification may be a sort of apathetic learning because it does not endeavor to build a common inner show, but basically stores occurrences of the preparing information. Classification is computed from a straightforward lion's share vote of the k closest neighbors of each point.

Advantages: This calculation is straightforward to actualize, vigorous to loud preparing information, and compelling in the event that preparing information is huge.

Disadvantages: Ought to decide the value of $\mathrm{K}$ and the computation fetched is tall because it should computer the remove of each occurrence to all the preparing tests.

\subsubsection{Random Forest}

Definition: Random forest classifier may be a meta-estimator that fits a number of choice trees on different sub-samples of datasets and employments normal to move forward the prescient precision of the show and controls over-fitting. The sub-sample size is continuously the same as the initial input test estimate, but the tests are drawn with replacement.

Advantages: Reduction in over-fitting and random forest classifier is more accurate than decision trees in most cases.

Disadvantages: Moderate genuine time forecast, troublesome to execute, and complex calculation.

\subsubsection{Support Vector Machine}

Definition: Support vector machine may be a representation of the preparing information as focuses in space separated into categories by a clear hole that's as wide as conceivable. Unused illustrations are at that point mapped into that same space and anticipated to have a place to a category based on which side of the crevice they drop.

Advantages: Successful in high dimensional spaces and employments a subset of preparing focuses within the decision function so it is additionally memory productive.

Disadvantages: The calculation does not specifically give likelihood gauges, these are calculated utilizing a costly fivefold cross-validation.

\section{PROPOSED SYSTEM}

The architecture of proposed system, represented in Figure 1, explains the overall process of Prediction. The prediction model uses Ensemble Learning which is a combination of various algorithms. The algorithm gets the data from an IoT setup which by considering various parameters like temperature, humidity, fire and gas concentrations. The model consists of 3 phases:

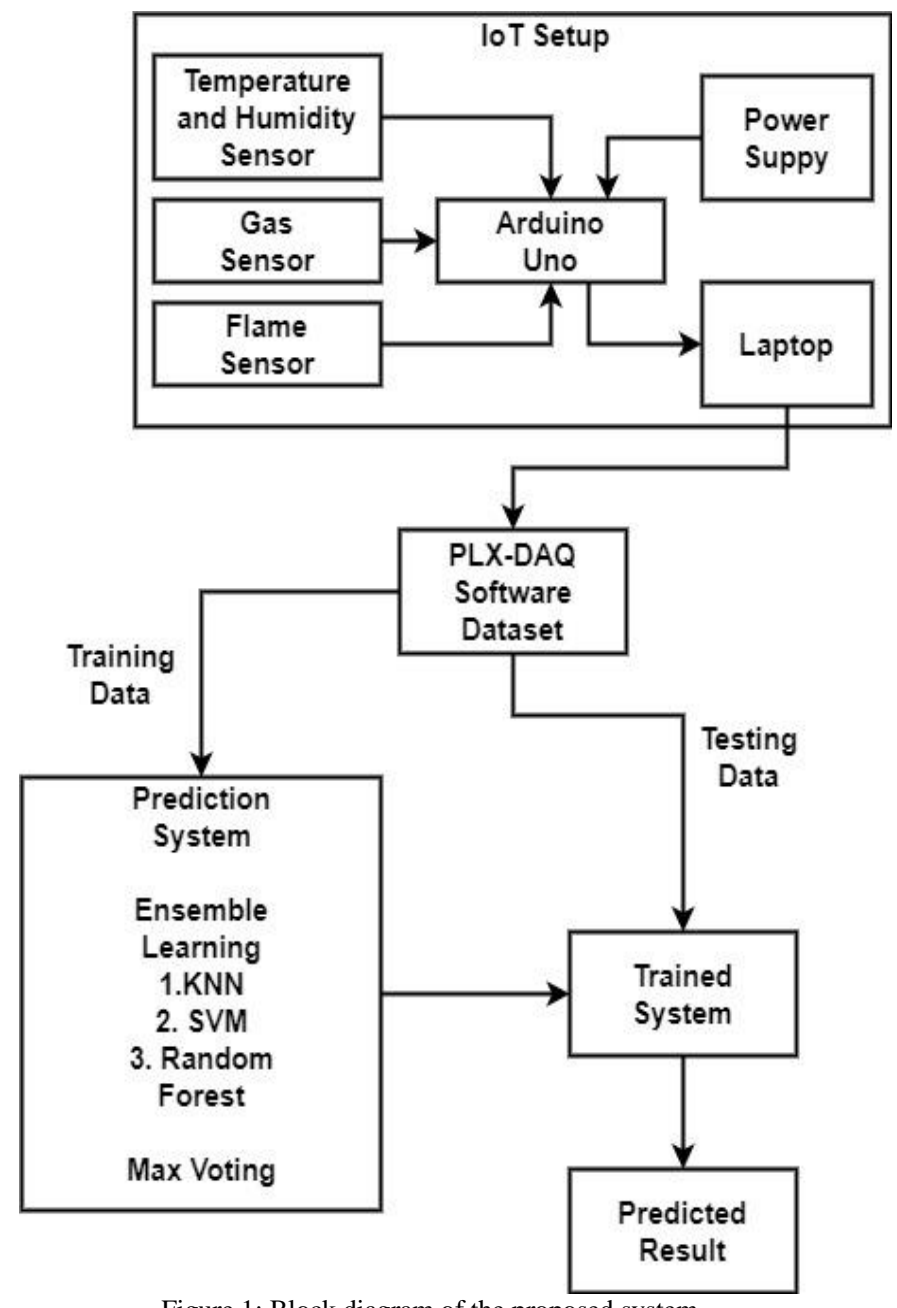

Figure 1: Block diagram of the proposed system

\section{A. Coal Mine Data Extraction}

The blasts in coal mines happen basically due to anomalies in temperature. So, we ought to keep a track on it which may increment due to nearness of hurtful vaporous particles interior the mines or due to fire breakouts. The highlights of the information such as temperature, mugginess, gas and fire are extricated from the Arduino Uno which is associated to different sensors. The samples are collected in the .csv record format employing the PLX-DAQ software program. This program makes a difference us in putting away the information in an organized way conjointly store it in a suitable organize, so that the information is prepared preparing the expectation model.

\section{B. Training Phase}

The information is collected in different situations by considering the destructive conditions that can happen interior the mines which may cause mishaps conjointly all conceivable combinations of temperature, stickiness, gas and fire are considered. The collected information is partitioned into preparing set and testing set. The training set of the collected information is given to the ensemble Learning Expectation 
Framework, which takes after a max voting strategy in choosing the result.

\section{Testing Phase}

After the Demonstrate is prepared, the Ensemble Learning Framework will get it how the conditions happen interior the mines, and what conditions contribute for a mishap. The endmost result is given after obtaining results from the classification of the sub level classifiers such as K Nearest Neighbor, Support Vector Machine Conjointly Random Forest. The forecast gotten could be a result of max voting that happens between the results gotten by these calculations.

\section{RESULTS}

\subsection{Connections}

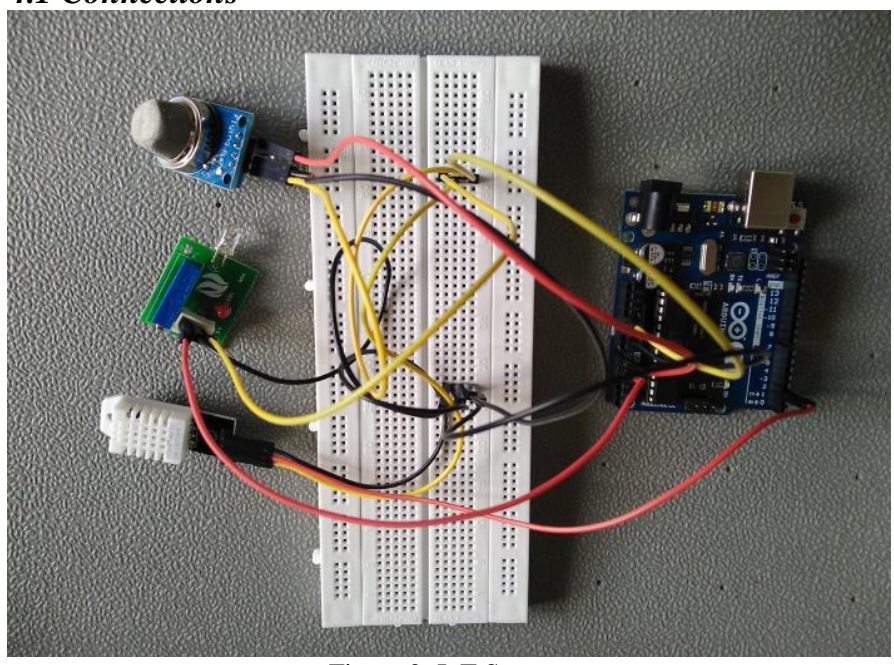

Figure 2: IoT Setup

The IoT Setup comprises of different sensors such as temperature, humidity, gas and fire which is associated to the Arduino uno through the breadboard as appeared in Figure 2. The VCC of all the sensors are associated and shorted on the breadboard. Additionally, all the grounds of the sensors are moreover shorted. The yields of the sensors are associated to analog pins of Arduino.

The data is collected considering the diverse conditions that can happen insides the coal mines. Circumstances are shifted to cover all viable states. The higher temperature readings are gotten by giving extra warm by implies of a heater and lower ones are recorded by utilizing a cooler or an ice cube. To encourage fire breakout examining we have utilized a candle. To supply the vaporous particles for the gas sensor, here we have utilized a joss stick.

\begin{tabular}{|c|c|c|c|c|c|c|}
\hline 1 & Time & Temperature & Gas & Fire & Humidity & Class \\
\hline 2 & $18: 15: 42$ & 37.6 & 1.37 & 197 & 41.7 & Normal \\
\hline 3 & $18: 15: 46$ & 37.6 & 1.41 & 197 & 40.4 & Normal \\
\hline 4 & $18: 17: 12$ & 36 & 2.54 & 198 & 39 & Warning \\
\hline 5 & $18: 17: 16$ & 36 & 2.8 & 197 & 39.3 & Warning \\
\hline 6 & $18: 17: 20$ & 35.9 & 3.09 & 198 & 39.3 & Danger \\
\hline 7 & $18: 17: 50$ & 35.4 & 3.05 & 198 & 39.9 & Danger \\
\hline 8 & $18: 17: 52$ & 35.4 & 2.98 & 197 & 40 & Warning \\
\hline 9 & $18: 17: 56$ & 35.3 & 2.18 & 197 & 40.5 & Warning \\
\hline 10 & $18: 18: 02$ & 35.2 & 1.96 & 197 & 40.6 & Normal \\
\hline 11 & $18: 18: 16$ & 36.3 & 1.71 & 198 & 40.2 & Normal \\
\hline 12 & $18: 18: 19$ & 37.3 & 1.69 & 198 & 40 & Warning \\
\hline 13 & $18: 18: 37$ & 46.6 & 1.62 & 198 & 33 & Warning \\
\hline 14 & $18: 18: 39$ & 47 & 1.6 & 198 & 32.1 & Warning \\
\hline 15 & $18: 18: 45$ & 47.9 & 1.56 & 198 & 29.6 & Danger \\
\hline 16 & $18: 18: 59$ & 48.1 & 1.5 & 198 & 25.7 & Danger \\
\hline 17 & 18:07:16 & 30.4 & 1.2 & 201 & 49.1 & Normal \\
\hline 18 & $18: 07: 18$ & 30.5 & 1.2 & 199 & 49.1 & Normal \\
\hline 19 & $18: 07: 30$ & 30.4 & 1.19 & 642 & 48.9 & Warning \\
\hline 20 & $18: 07: 32$ & 30.4 & 1.19 & 702 & 48.9 & Warning \\
\hline 21 & $18: 07: 34$ & 30.4 & 1.19 & 1020 & 48.9 & Danger \\
\hline 22 & $18: 07: 38$ & 30.4 & 1.2 & 1020 & 48.9 & Danger \\
\hline
\end{tabular}

The datasets collected from the over stages is shown within the Figure 3. The above shown datasets is one of the samples from entire training data. It consists of time, temperature, gas, fire, humidity and class as the fields. Here time is considered as the PLX-DAQ accepts the data in the same format which is preprogrammed in the software. All other fields act as a deciding factor to determine which class the test set may belong to between the normal, warning and danger.

\subsection{Result of Training the Model}

The training set is passed to python program to form the prediction system representation as shown in figure 4 . The result is put away in a .csv format file. This model will be read and used to check the working of the model from the testing. After the forecast, the result looks as appeared within the figure underneath. 


$$
\begin{aligned}
& 29.40,1.72,954.00,53.70 \Rightarrow \text { Danger } \\
& 29.30,1.73,953.00,53.90 \Rightarrow \text { Danger } \\
& 29.30,1.78,954.00,54.10 \Rightarrow \text { Danger } \\
& 29.30,1.81,954.00,54.20 \Rightarrow \text { Danger } \\
& 29.30,1.84,264.00,54.30 \Rightarrow \text { Normal } \\
& 29.20,1.86,265.00,54.30 \Rightarrow \text { Normal } \\
& 29.30,1.87,264.00,54.40 \Rightarrow \text { Normal } \\
& 29.20,1.89,266.00,54.40 \Rightarrow \text { Normal } \\
& 29.20,1.90,953.00,54.40 \Rightarrow \text { Danger } \\
& 29.30,1.91,265.00,54.50 \Rightarrow \text { Normal } \\
& 29.30,1.93,265.00,54.60 \Rightarrow \text { Normal } \\
& 29.20,1.94,953.00,54.60 \Rightarrow \text { Danger } \\
& 29.20,1.94,264.00,54.60 \Rightarrow \text { Normal } \\
& 29.30,1.95,264.00,54.70 \Rightarrow \text { Normal } \\
& 29.20,1.96,952.00,54.70 \Rightarrow \text { Danger } \\
& 29.30,1.97,953.00,54.80 \Rightarrow \text { Danger } \\
& 29.20,1.97,953.00,54.70 \Rightarrow \text { Danger } \\
& 29.20,1.97,261.00,54.80 \Rightarrow \text { Normal } \\
& 29.30,1.98,261.00,54.90 \Rightarrow \text { Normal } \\
& 29.30,1.98,259.00,54.90 \Rightarrow \text { Danger } \\
& 29.30,1.99,260.00,54.70 \Rightarrow \text { Normal } \\
& 29.30,2.05,275.00,54.70 \Rightarrow \text { Warning } \\
& 29.30,2.06,278.00,54.70 \Rightarrow \text { Warning } \\
& 29.30,2.02,271.00,54.70 \Rightarrow \text { Warning } \\
& 29.30,1.99,257.00,54.60 \Rightarrow \text { Danger } \\
& 29.30,2.03,264.00,54.60 \Rightarrow \text { Danger } \\
& 29.40,2.02,258.00,54.60 \Rightarrow \text { Warning } \\
& 29.40,2.05,268.00,54.50 \Rightarrow \text { Warning } \\
& 29.40,2.03,257.00,54.40 \Rightarrow \text { Warning }
\end{aligned}
$$

Figure 4: Testing Output

The values in the row represent the sensor values of Temperature, Gas , Fire and Humidity from left to right followed by the predicted class for the particular instance. The samples for which the predicted value is Normal are displayed in green colour. Similarly for the samples having predicted class as Warning and Danger are displayed in magenta and red respectively.

\subsection{Result of Performance Evaluation}

Accuracy, recall, precision and $\mathrm{f} 1$ score are measured for the trained model and are implemented for the set of testing samples is shown in Figure 5 along with confusion matrix and classification report.

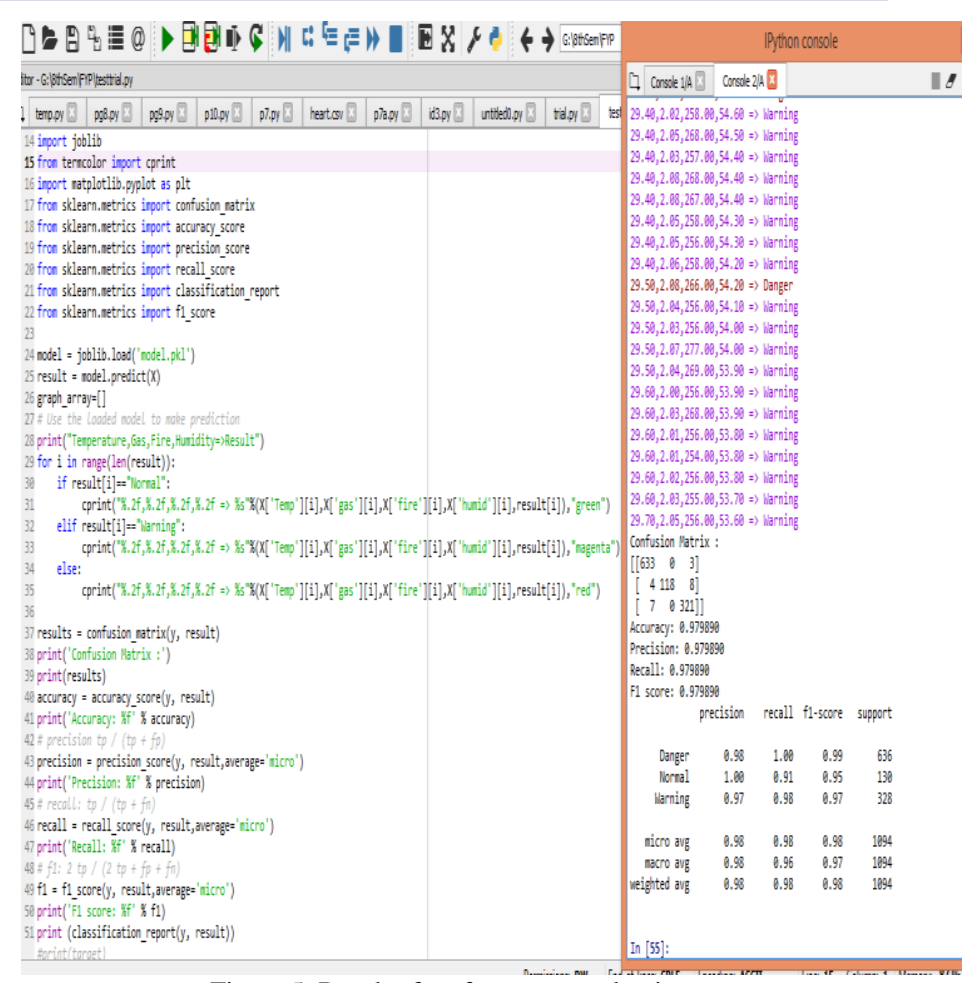

Figure 5: Result of performance evaluation

\section{CONCLUSIONS}

Coal Mine Calamity Prediction utilizing IoT and Machine Learning is displayed, which could be a forecast framework that can bring the persistent genuine time information from the coal mine environment and predicts whether there's reasonable condition for mineworkers to work. IoT setup is outlined to accumulate the information by considering different parameters such as temperature, mugginess, gas, and fire anomalies which are primary reasons for blasts and roof falls inside the mines. Besides, for the machine learning perspective of the project we are utilizing ensemble learning calculation for forecast which may be a combination of various calculations like K Neighbors Classifier, Random forest and Support Vector Machine. As this application employments IoT, there's an arrangement to encourage ceaseless genuine time information to anticipate and see the leads to genuine time utilizing model form of all the highlights in honest to goodness time utilizing demonstrate adjustments of all the highlights and not fair utilizing the available data to anticipate, which makes the work also challenging.

There are numerous upgrades that can be nonetheless made. This extend can be amplified to other areas such as discuss contamination expectation system, forest fire forecast frameworks, etc. The framework can too center on coordinate contact with the diggers by giving gadgets such as head protectors, watch, etc. that can be closer to the mineworkers and caution them around the threat.

\section{REFERENCES}

[1] S.Sindhu, J.Subashree, J.Suganthi, M.Vinodhini, R. Umamaheswari, "Coal Mining Safety using IoT", Imperial Journal of Interdisciplinary Research (IJIR) Vol-3, Issue-3, 2017.

[2] Mr.Arun Katara, Anand Dandale, Abhilesh Chore,Anurag Bhandarwar, "ZigBee Based Intelligent Helmet for Coal Miners",IEEE, 31 March-2 April, 2009. 
[3] Arnab Kumar Saha, Sachet Sircar, Priyasha Chatterjee, Souvik Dutta, Anwesha Mitra, "A raspberry Pi controlled cloud based air and sound pollution monitoring system with temperature and humidity sensing”,IEEE, 8-10 Jan, 2018.

[4] Mr. Onkar Amale, Dr. Rupali Patil; Electronics \& Telecomm Department, "IOT Based Rainfall Monitoring System Using WSN Enabled Architecture", IEEE, 27-29 March, 2019.

[5] Desheng Liu, Zhiru Xu, Wei Wang, Lei Wang, "Prediction of Coal Mine Safety Level Based on LSSVM", IEEE, 24-25 April ,2010.

[6] Yuping Wu, "Application of Support Vector Machine in Coal and Gas Outburst Area Prediction",IEEE, 20-22 Nov, 2009.

[7] Wang Heng, Shao Liangshan, Liu Shuanhong, Lu Lin, "Prediction of Coal/Gas Outbursts based on selective ensemble learning", IEEE, 16-18 Jan, 2013.

[8] Jinpeng Li, Li Gao,"Information Fusion Technology Based on T-S Fuzzy Neural Network for Coal Mine Gas Prediction",IEEE, 21-25 Oct, 2012.

[9] Wikipedia,https://en.wikipedia.org/wiki/1965_Dhanbad_coal_mine_dis aster

[10] Wikipedia,https://en.wikipedia.org/wiki/2018_Meghalaya_mining_acci dent

[11] Wikipedia,https://en.wikipedia.org/wiki/Chasnala_mining_disaster 